\section{Análise clínica e epidemiológica das internações hospitalares de idosos decorrentes de intoxicações e efeitos adversos de medicamentos, Brasil, de 2004 a 2008}

\section{Clinical and epidemiological analysis of hospitalizations of elderly due to poisoning and adverse effects of medications, Brazil from 2004 to 2008}

\section{Tatiana Cruz de Paula'}

\section{Rosany Bochner"}

\section{Dalia Elena Romero Montilla"}

' Programa Institucional de Bolsas de Iniciação Científica do CNPq / Universidade Federal Fluminense (UFF). Instituto de Comunicação e Informação Científica e Tecnológica em Saúde (ICICT) da Fundação Oswaldo Cruz.

"Instituto de Comunicação e Informação Científica e Tecnológica em Saúde (ICICT) da Fundação Oswaldo Cruz - FIOCRUZ, RJ.

Fonte financiadora: CNPq/FIOCRUZ.

Correspondência: Rosany Bochner. Fundação Oswaldo Cruz. Av. Brasil, 4.365 - Prédio Haity Moussatché, $2^{\circ}$ andar, sala 206, Rio de Janeiro RJ CEP 21045-900 . E-mail: rosany@icict.fiocruz.br

\section{Resumo}

Os idosos são mais suscetíveis aos efeitos adversos dos medicamentos devido a uma diversidade de fatores, tais como o uso excessivo e concomitante de diversos fármacos, erros de administração, alterações fisiológicas no organismo que alteram a farmacodinâmica e a farmacocinética. Com o objetivo de determinar as principais classes terapêuticas envolvidas nas internações hospitalares de idosos decorrentes de intoxicação e efeito adverso de medicamentos, bem como os principais agravos relacionados a esses eventos, foram analisadas 9.793 internações hospitalares de pessoas com 60 anos ou mais, registradas no Brasil pelo Sistema de Internações Hospitalares do SUS (SIH-SUS), no período de 2004 a 2008. Medicamentos não especificados, antibióticos sistêmicos, psicotrópicos, psicoativos, antiepilépticos, sedativos, hipnóticos e antiparkinsonianos, responderam por $57 \%$ do total de internações analisadas. Traumatismos e quedas foram os principais agravos relacionados a intoxicação e efeito adverso de medicamentos. As internações por traumatismo apresentaram relação com analgésicos, antitérmicos e antirreumáticos não opiáceos. Já as quedas estavam associadas aos antibióticos sistêmicos, contrariando os estudos que apontam os psicotrópicos como os principais medicamentos envolvidos nesses eventos. Os resultados encontrados refletem a tendência crescente dos problemas associados ao uso de medicamentos por idosos. Verificou-se que o perfil de consumo não é suficiente para explicar as concentrações de casos nas principais classes terapêuticas. Na busca pelas causas, sugere-se a adoção de programas mais efetivos de farmacovigilância, capazes de monitorar as diferentes etapas do processo de uso de medicamentos: prescrição, dispensação, comercialização, administração e adesão ao tratamento.

Palavras-chave: Idosos. Intoxicações. Internações. Classes terapêuticas. Fraturas. Quedas. 


\section{Abstract}

The elderly are more susceptible to adverse drugs effects due to a variety of factors, such as excessive and concomitant use of several drugs, administration errors, physiological changes in the body that alter the pharmacodynamics and pharmacokinetics. In order to determine the main therapeutic classes involved in hospital admissions of elderly people due to intoxication and adverse drug effects, as well as major health problems related to these events, 9,793 hospitalizations of people aged 60 or over registered in the Hospital Information System of the Unified Health System (SIH-SUS) in the period of 2004 to 2008 were analyzed. Unspecified drugs, systemic antibiotics, psychotropics, psychoactives, antiepileptics, sedatives, hypnotics and antiparkinsonians accounted for $57 \%$ of the total of admissions analyzed. Injuries and falls were the main health problems related to intoxication and adverse drug effects. Hospitalizations due to injuries were associated with analgesic, antipyretic and antirheumatic non-opioid. Falls were associated with systemic antibiotics, contradicting studies which point out psychotropic drugs as the main drug involved in these events. The results reflect the growing trend of problems associated with drug use by elderly people. It was verified that the consumption profile alone is not sufficient to explain the concentration of cases of the major therapeutic classes. We suggest the adoption of more effective programs of pharmacovigilance, capable of intervening at different stages of drug use: prescribing, dispensing, marketing, administration and compliance.

Keywords: Elderly. Intoxications. Hospitalizations. Therapeutic classes. Fractures. Falls.

\section{Introdução}

A população brasileira vem envelhecendo. Se, em 1980 os idosos, indivíduos com idade igual ou superior a 60 anos ${ }^{1}$, representavam $6 \%$ da população, em 2010 já somavam $11 \%^{2}$, e para 2025 estima-se que alcancem $15 \%$, fazendo com que o Brasil apresente a $6^{\text {a }}$ maior população de idosos do mundo ${ }^{3,4}$.

O processo natural de envelhecimento gera modificações estruturais e funcionais no organismo humano, e tem como consequência direta um aumento na prevalência de doenças características dos idosos. Nesta faixa etária observa-se alta prevalência de doenças crônicas, principalmente as neurodegenerativas, psiquiátricas, cardiovasculares e metabólicas ${ }^{5,6}$.

Uma vez que os idosos se encontram mais propensos a apresentar um número maior de doenças, estão expostos a um maior consumo de medicamentos ${ }^{7-12} \mathrm{e}$, consequentemente, aos riscos de sua utilização excessiva. Além disso, as mudanças características do envelhecimento, que podem comprometer a ação e a metabolização dos fármacos no organismo, somadas à falta de conhecimento a respeito da eficácia e da segurança de muitos medicamentos para o organismo delicado dos idosos, aumentam a probabilidade de ocorrência de intoxicações e efeitos adversos de medicamentos nessa faixa etária ${ }^{13,14}$.

Em princípio, dados de intoxicação registrados no país pelo Sistema Nacional de Informações Tóxico-Farmacológicas (SINITOX) ${ }^{15}$ poderiam contradizer os achados anteriores, uma vez que ao calcular os coeficientes de morbidade para os idosos obtêm-se os menores valores comparados às demais faixas etárias. Contudo, se não chamam a atenção pelo número de casos, apresentam os maiores coeficientes de mortalidade e de letalidade, o que seria um indicador da maior gravidade desses eventos para os idosos.

A maior suscetibilidade da população idosa às reações adversas mais graves de medicamentos pode ser observada em 
diferentes estudos internacionais que, baseados em dados de internações hospitalares por esse agravo, verificaram maior frequência nessa faixa etária ${ }^{16-21}$. Em um estudo de metanálise baseado em 64 artigos concluiu-se que os idosos possuem uma chance quatro vezes maior de serem hospitalizados por problemas relacionados a reações adversas de medicamentos do que o restante da população ${ }^{22}$.

Dada a importância das reações adversas nessa faixa etária, trabalhos de diferentes países passaram a focalizar o agravo no paciente idoso hospitalizado. Alguns estudos consideraram apenas as reações adversas, ou seja, os efeitos indesejados provenientes do uso correto dos medicamentos $^{23-25}$, e mostraram que há um grande número de reações adversas graves, mesmo quando os medicamentos são corretamente prescritos e administrados. Outros trabalhos utilizaram um conceito mais amplo, o de evento adverso, no qual os erros de medicação (qualquer erro no processo de prescrição, transcrição, dispensação, administração e monitoramento do tratamento ${ }^{26}$ eram também contemplados ${ }^{26-32}$ O caráter evitável desses eventos tem sido abordado por vários autores ${ }^{19,26,28-31}$. Há quem afirme que boa parte das reações adversas pode ser prevenida por meio de melhorias simples no processo de prescrição (revisão periódica das receitas, uso de prescrição informatizada e participação do farmacêutico na avaliação da prescrição), uma vez que as interações medicamentosas estavam presentes em quase $17 \%$ desses eventos $^{20,31}$. Outros apontaram para problemas relacionados com a adesão do paciente ao tratamento e à automedicação ${ }^{26}$. Contudo, o mais surpreendente foi verificar que os medicamentos mais envolvidos nas internações de idosos decorrentes de eventos adversos eram comumente os utilizados por essa população e não aqueles considerados de maior risco ${ }^{20,27-30}$.

No Brasil há ainda um longo percurso a percorrer. Já foram realizados estudos baseados no banco de dados hospitalares de abrangência nacional, o Sistema de
Informações Hospitalares do SUS (SIHSUS), para estudar a morbidade por lesões e envenenamentos no país ${ }^{33}$, causas externas $^{34}$ e em geral ${ }^{35}$ entre idosos no Brasil, os agravos provocados por medicamentos em hospitais do Rio de Janeiro ${ }^{36}$ e as intoxicações e efeitos adversos de medicamentos em crianças menores de um ano no Brasil ${ }^{37}$. Trabalhos mais específicos, abordando idosos internados acometidos por intoxicações e reações adversas de medicamentos foram realizados apenas em âmbito local, com base em alguns hospitais ${ }^{38,39}$.

Dessa forma, com o objetivo de ampliar o conhecimento sobre o impacto do uso de medicamentos pela população idosa, foram analisadas as internações hospitalares de indivíduos com 60 anos ou mais relacionadas à intoxicação e efeito adverso de medicamentos registradas no país, identificando as principais classes terapêuticas e agravos envolvidos.

\section{Metodologia}

O desenho de estudo é retrospectivo, e abrange as internações hospitalares registradas em todo o território brasileiro pelo Sistema de Informações Hospitalares do SUS (SIH-SUS) entre 2004 e 2008, sendo empregados como fonte de dados os CDROMs do Movimento de Autorização de Internação Hospitalar do SUS ${ }^{40-44}$.

A população da pesquisa é formada por idosos que, de acordo com o Estatuto do Idoso, lei $\mathrm{n}^{\circ} 10.741$ de $1^{\circ}$ de outubro de $2003^{1}$, abrange pessoas com idade igual ou superior a 60 anos.

$\mathrm{Na} 10^{a}$ Revisão da Classificação Internacional de Doenças (CID-10) ${ }^{45}$ há códigos que identificam tanto os efeitos adversos quanto as intoxicações por medicamentos. Segundo a Organização Mundial da Saúde ${ }^{46}$, a reação adversa a medicamentos é definida como qualquer efeito nocivo, não intencional e indesejável de um medicamento, que ocorre em doses usadas em seres humanos para profilaxia, diagnóstico ou terapia. Na CID- $10^{45}$, essa definição é adotada para efeito adverso de 
medicamentos e os códigos referentes são: T80.5, T80.6, T88.6, T88.7, Y40 a Y57. Com relação às intoxicações por medicamentos, os códigos correspondentes da CID-10 são: F11.0, F13.0, F15.0, F19.0, F55, T36-T39, T40.2, T40.3, T40.4, T41-T50, X40, X41, X43, X44, X60, X61, X63, X64, X85, Y10, Y11, Y13, Y14, que englobam falhas terapêuticas, eventos acidentais e intencionais, abuso e demais problemas relacionados ao uso indevido ou não racional de medicamentos.

Foram considerados "casos" as internações de idosos que apresentaram, no diagnóstico principal e/ou no diagnóstico secundário, um dos códigos definidos para intoxicações ou efeitos adversos de medicamentos. Salienta-se que esses mesmos códigos foram adotados por Lessa et al. ${ }^{37}$ em seu estudo sobre internações de crianças menores de 1 ano de idade relacionadas a intoxicações e efeitos adversos de medicamentos.

Assim como foi adotado por Rozenfeld ${ }^{36}$ e Lessa et al. ${ }^{37}$, os códigos X42, X62 e Y12 foram suprimidos a fim de evitar a seleção de códigos da CID- $10^{45}$ que não permitissem diferenciar as intoxicações associadas ao uso de medicamentos daquelas associadas ao uso de drogas lícitas e ilícitas.

De acordo com a metodologia proposta por Lessa et al. ${ }^{37}$, foram consideradas quatro situações, resultantes de combinações entre os diagnósticos principal e secundário. A situação 1 identifica internações nas quais a intoxicação ou o efeito adverso de medicamentos, ou seja, o evento de interesse, aparece no diagnóstico secundário e não no principal. Essa situação é a única onde o evento de interesse não constitui o motivo da internação, podendo, no entanto, ter iniciado a cadeia de acontecimentos patológicos que conduziram diretamente ao diagnóstico principal ${ }^{47}$. Pode ocorrer também do evento de interesse se desenvolver durante a internação, afetando as condições do paciente ${ }^{48}$. A situação 2 apresenta a intoxicação ou efeito adverso de medicamentos no diagnóstico principal, ficando para o diagnóstico secundário os demais agravos. As situações 1 e 2 permitem analisar os principais agravos relacionados à intoxicação ou o efeito adverso de medicamentos. A situação 3 traz tanto no diagnóstico principal quanto no secundário códigos relacionados a intoxicação ou efeito adverso de medicamentos. Essa situação é a que, teoricamente, deveria conter mais informações sobre o evento de interesse, em especial sobre a circunstância em que esse ocorreu. A situação 4 só apresenta o diagnóstico principal preenchido por intoxicação ou efeito adverso de medicamentos, ficando o diagnóstico secundário em branco.

Para as internações relacionadas à intoxicação, pode-se determinar a circunstância associada com base nos códigos do capítulo 20 da CID- $10^{45}$. Os códigos X40-X44 referem-se a circunstância acidental; X60-X64 a auto-intoxicação; X85 a agressão e Y10-Y14 a não determinada. Dessa forma, apenas as internações relacionadas à intoxicação que tiveram seu diagnóstico principal ou secundário definidos por esses códigos puderam ter sua circunstância descrita por uma das categorias apresentadas. Para as demais, foi assumida a circunstância ignorada.

Um maior detalhamento das classes terapêuticas envolvidas nos casos de intoxicação e efeito adverso de medicamentos pôde ser realizado para as internações em que o diagnóstico principal ou secundário tenha sido preenchido com os quatro dígitos previstos para os códigos do capítulo $19 \mathrm{e}$ para os agrupamentos $\mathrm{Y} 40$ a Y57 do capítulo 20 da CID- $10^{45}$. Contudo, pelo grande uso dos dígitos 8 e 9 , relacionados às categorias "outras" e "não especificadas", os resultados encontrados para a maioria das substâncias não são representativos. Para a categoria T50 foi importante considerar o quarto dígito para separar os casos de intoxicação por outras drogas e as não especificadas (T50.9) daqueles referentes aos diuréticos.

Os dados populacionais, referentes aos anos de 2004 a 2008, foram obtidos das estimativas do IBGE divulgadas pelo Datasus ${ }^{2} \mathrm{e}$ utilizados para o cálculo das taxas de internação hospitalar por 100 mil habitantes por classificação do caso (intoxicação ou efeito 
adverso de medicamentos), por sexo e para cada um dos grupos etários, 60 a 64, 65 a 69, 70 a 74,75 a 79 e 80 anos e mais.

Para identificar os agravos relacionados com as intoxicações e efeitos adversos de medicamentos, foram analisados os diagnósticos principais envolvidos com a situação 1 e os diagnósticos secundários envolvidos com a situação 2 .

Para testar a independência entre as especialidades do atendimento, entre os sexos, entre as faixas etárias, bem como entre as classes terapêuticas e as variáveis situação, classificação do caso e circunstância da intoxicação, foi aplicado o teste qui-quadrado, com nível de significância de $5 \%{ }^{49}$.

Para testar se há diferença entre as situações, bem como entre as circunstâncias da intoxicação, com base nas idades e no tempo de permanência no hospital, foi aplicado o teste não paramétrico de Kruskal-Wallis, uma vez que as suposições de normalidade e homogeneidade de variâncias não foram atendidas, exigências estas necessárias para a aplicação de um teste paramétrico mais poderoso e conhecido, Análise deVariância $\left(\right.$ ANOVA) ${ }^{49}$.

Para testar se há diferença entre os sexos e entre a classificação dos casos em "intoxicação" ou "efeito adverso", com base nas idades e no tempo de permanência no hospital, foi aplicado o teste não paramétrico de Wilcoxon-Mann-Whitney, uma vez que as suposições de normalidade e homogeneidade de variâncias não foram atendidas, exigências estas para a aplicação do teste paramétrico $t^{49}$.

O projeto foi aprovado pelo Comitê de Ética em Pesquisa em Seres Humanos do Instituto de Pesquisa Clínica Evandro Chagas (IPEC) da Fundação Oswaldo Cruz (Fiocruz), com o parecer n 020/2009.

\section{Resultados}

No Brasil, no período de 2004 a 2008, foram registradas pelo Sistema de Internações Hospitalares do SUS (SIH-SUS), 9.793 internações de idosos relacionadas a intoxicações e efeitos adversos de medicamentos. Nesse período, esses eventos apresentaram um crescimento de $13,6 \%$, passando de 1.857 casos em 2004 para 2.109 em 2008. O número de internações relacionado a intoxicações cresceu em todas as faixas etárias de forma bastante diferenciada, variando de 9,9\% para os idosos com 80 anos e mais até $88,7 \%$ para os de 60 a 64 anos. Por outro lado, para efeitos adversos o número de internações diminuiu em todos os grupos etários de forma mais homogênea, variando de $44,7 \%$ para os idosos de 80 anos e mais até $61,1 \%$ para os de 70 a 74 anos.

A Figura 1 mostra tendência decrescente para todas as faixas etárias em se tratando de efeito adverso, sendo crescente nos casos de intoxicação para as faixas de 60 a 64, 65 a 69 e de 70 a 74 anos. Verifica-se que as taxas de internação hospitalar aumentam com o avanço da idade, tanto para os casos de intoxicação quanto para os de efeito adverso, exceção esta observada nos anos de 2007 e 2008 para as intoxicações, nos quais as taxa dos idosos de 60 a 64 anos $(7,08$ e 9,75) mostrou-se superior a dos de 65 a 69 anos (6,93 e 8,33), e no ano de 2004 para os efeitos adversos, quando a taxa dos idosos de 75 a 79 anos $(4,95)$ foi muito próxima daquela dos de 80 anos e mais $(4,91)$.

A partir dos dados da Tabela 1 pode-se constatar que as quatro situações, criadas a partir da combinação dos diagnósticos, diferem significativamente quanto às proporções de cada uma das especialidades do atendimento (teste qui-quadrado, $\mathrm{p}$-valor $=0$ ). $O$ percentual referente à cirurgia para a situação 1 chega a $48,6 \%$, enquanto que para as demais situações não chega a $0,6 \%$. Por outro lado, a situação 4 apresenta 17,3\% de internações na especialidade psiquiatria, enquanto que para as demais situações essa categoria não alcança $0,2 \%$. As faixas etárias referentes aos mais jovens, 60 a 64 anos, e aos mais velhos, 80 anos e mais, apresentaram as maiores concentrações de casos, $24,4 \%$ e $21,5 \%$, respectivamente. As quatro situações diferem significativamente quanto às proporções das faixas etárias (teste qui-quadrado, $p$-valor $=2,22389 \mathrm{E}-23)$. A 


\section{Intoxicação}

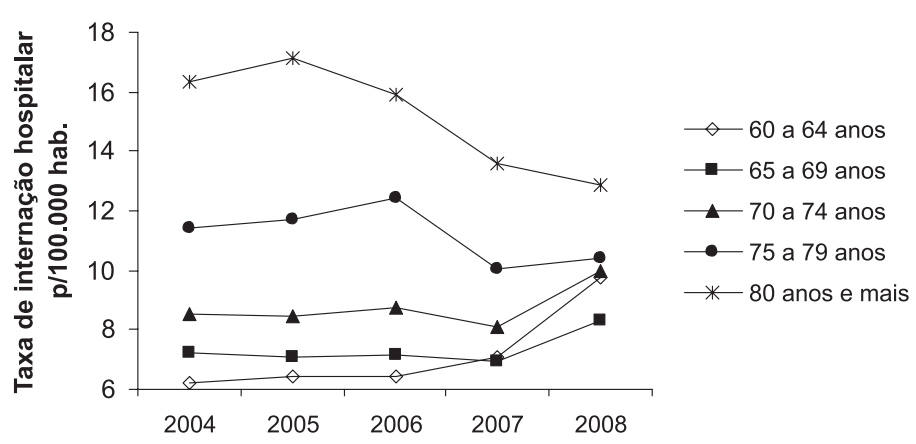

\section{Efeito Adverso}

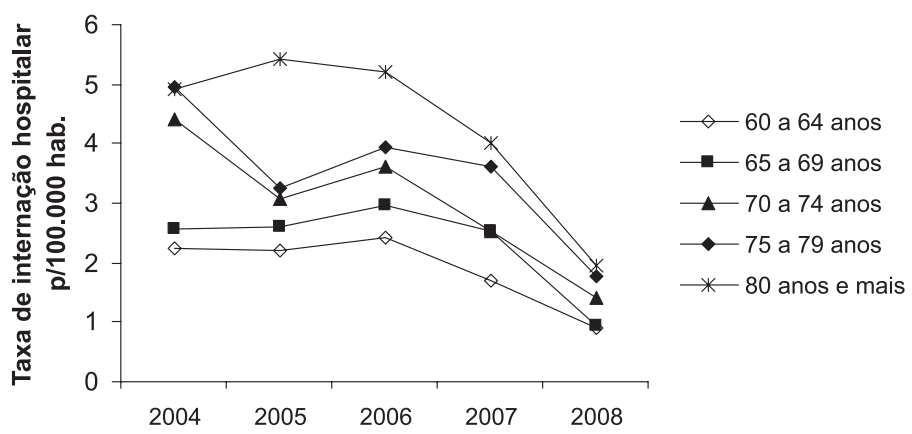

Fonte/Source: SIH-SUS

Figura 1 - Taxas de internação hospitalar por 100.000 hab. relacionadas a intoxicação e efeito adverso de medicamentos distribuídas por faixa etária. Brasil, 2004 a 2008.

Figure 1 - Rates of hospitalization per 100,000 inhabitants related to drug intoxication and adverse effects distributed by age group. Brazil, 2004 to 2008.

situação 4 apresenta a maior proporção de idosos com 60 a 64 anos (41,3\%) e a menor proporção de idosos com 80 anos e mais $(12,7 \%)$. Observando-se o total de internações, a população feminina contribuiu com o maior número absoluto e a masculina com a maior taxa de internação hospitalar, 12,11 e 11,41 por 100 mil habitantes, respectivamente. As quatro situações diferem significativamente quanto às proporções de cada sexo (teste qui-quadrado, p-valor $=9,43234$ E-37). As situações 1 e 4 apresentam proporções maiores para o sexo masculino (50,6\% e $66,4 \%$, respectivamente), enquanto que nas situações 2 e 3 o sexo feminino mostra-se mais frequente $(56,2 \%$ e $60,6 \%$, respectivamente). As taxas de internação hospitalar por faixa etária para o sexo masculino foram superiores as do feminino para os idosos de 60 a 64 anos (10,36 e 8,05); para os de 65 a 69 anos $(10,66$ e 8,78) e para os de 70 a 74 anos $(12,23$ e 11,29). A partir de 75 anos há uma inversão, e as taxas referentes ao sexo feminino superam as do masculino; na faixa de 75 a 79 anos tem-se 14,81 e 14,18, e para os com 80 anos e mais esta relação passa para 20,01 e 17,65 , respectivamente. Verificou-se também um número maior de casos de intoxicação por medicamentos $(77,3 \%)$ em comparação com os casos de efeito adverso $(22,7 \%)$. As quatro situações diferem significativamente quanto às proporções desses eventos (teste qui-quadrado, $\mathrm{p}$-valor $=0$ ). O percentual referente a efeito adverso de medicamentos, 
entre os casos relacionados à situação 1 , é praticamente o dobro do apresentado para o total dos casos $(42,6 \%$ e $22,7 \%$, respectivamente), enquanto que para as situações $2 \mathrm{e}$ 4 esse percentual não chega a $3 \%$ e, para a situação 3 , fica em torno do valor geral, $27,2 \%$. A circunstância da intoxicação na maioria das vezes é ignorada (51,0\%), seguida pelas acidentais (17,3\%), autointoxicação (15,6\%) e não determinada $(14,6 \%)$. As agressões foram responsáveis por apenas $1,5 \%$ dos casos de intoxicação. As quatro situações diferem significativamente quanto às proporções desses eventos (teste qui-quadrado, p-valor $=0$ ). As situações 2 e 4 praticamente não discriminam as circunstâncias das intoxicações; a situação 3 apresenta maior percentual para a circunstância acidental (45,6\%) e a situação 1 aponta a autointoxicação como a mais frequente $(33,7 \%)$. Quanto às principais classes terapêuticas envolvidas nas internações de idosos, outros medicamentos e os não especificados foram responsáveis pelo maior número de casos, com $24,0 \%$ das internações; os antibióticos sistêmicos aparecem em segundo lugar, com $17,0 \%$, seguidos pelos psicotrópicos, psicoativos, antiepilépticos, sedativos, hipnóticos e antiparkinsonianos, com 16,2\% de participação. Esses três grupos respondem por $57,2 \%$ do total de internações analisadas. As quatro situações diferem significativamente quanto às proporções das classes terapêuticas (teste qui-quadrado, $\mathrm{p}$-valor $=0$ ). Na situação 1 , destacam-se os analgésicos, antitérmicos e anti-reumáticos não opiáceos com $25,4 \%$ de participação. Na situação 2 surgem os antibióticos sistêmicos com $32,4 \%$. Na situação 3 prevalecem os outros medicamentos e os não especificados com $27,7 \%$. Na situação 4 , grande concentração de casos deve-se aos psicotrópicos, psicoativos, antiepilépticos, sedativos, hipnóticos e antiparkinsonianos com $43,5 \%$.

Na Tabela 2 destacam-se para a situação 1 os traumatismos em geral, sendo os referentes aos membros inferiores os mais freqüentes (42\%). Com relação à situação 2 , as quedas concentram um terço das ocorrências, sendo a residência o local mais apontado para a ocorrência desses eventos (24\%). Dentre os traumatismos em geral, a participação do sexo feminino é superior ao do masculino, 59\%, sendo esta ainda maior para as fraturas do antebraço (76\%), do fêmur (69\%) e do ombro e braço (69\%).

De acordo com a Figura 2 pode-se verificar que os traumatismos e as quedas diferem significativamente quanto às proporções que apresentam das classes terapêuticas (teste qui-quadrado, $\mathrm{p}$-valor $=2,145 \mathrm{E}-270$ ) . Para os traumatismos, destacam-se os analgésicos, antitérmicos e anti-reumáticos não opiáceos (44,8\%), enquanto que para as quedas, a maior concentração de casos é associada aos antibióticos sistêmicos (37,8\%). A proporção de hormônios, seus substitutos sintéticos e seus antagonistas apresenta-se oito vezes maior para traumatismos em relação às quedas. Por outro lado, apesar de apresentarem valores inferiores a $10 \%$, a proporção de substâncias que atuam primariamente sobre o aparelho circulatório e sobre o aparelho cardiovascular, bem como a proporção de substâncias de ação no trato gastrointestinal referentes às quedas são dez e vinte e uma vezes maiores que as referentes aos traumatismos, respectivamente.

Na Figura 3 é possível observar que os antibióticos sistêmicos e os analgésicos apresentam participações bastante superiores nas internações relacionadas à intoxicação, $20 \%$ e $11 \%$, em comparação às relacionadas a efeito adverso, $7 \%$ e $5 \%$, respectivamente. Em contrapartida, para os hormônios, seus substitutos sintéticos e seus antagonistas, para as substâncias de ação essencialmente sistêmica e substâncias hematológicas e para os fármacos que afetam principalmente o sistema nervoso autônomo, ocorre o inverso, as internações relacionadas com efeito adverso apresentam percentuais superiores às da intoxicação, $12,3 \%, 10,7 \%$ e $10,6 \%$ contra $1,3 \%, 2,4 \%$ e $4,4 \%$, respectivamente. Com relação à circunstância da intoxicação, $41 \%$ das internações relacionadas a acidente foram causadas por outros medicamentos e os não especificados. Os analgésicos, antitérmicos e antirreumáticos não opiáceos 
Tabela 1 - Casos de internação hospitalar em idosos decorrentes de intoxicação e efeito adverso de medicamentos distribuídos por especialidade do atendimento, faixa etária, sexo, classificação do caso, circunstância da intoxicação e classe terapêutica segundo situação. Brasil, 2004 a 2008.

Table 1 - Cases of hospitalization related to drug intoxication and adverse effects in elderly distributed by specialty care, age group, sex, classification case, intoxication cause and therapeutic classes by situation. Brazil, 2004 to 2008.

\begin{tabular}{|c|c|c|c|c|c|}
\hline \multirow{3}{*}{ Variáveis } & \multicolumn{5}{|c|}{ Casos } \\
\hline & \multicolumn{4}{|c|}{ Situação } & \multirow{2}{*}{ Total } \\
\hline & 1 & 2 & 3 & 4 & \\
\hline \multicolumn{6}{|l|}{ Especialidade do atendimento } \\
\hline Cirurgia & 1664 & 7 & 1 & 3 & 1675 \\
\hline Clínica Médica & 1745 & 3250 & 2548 & 440 & 7983 \\
\hline Psiquiatria & 2 & 2 & 1 & 94 & 99 \\
\hline Outra & 13 & 14 & 4 & 5 & 36 \\
\hline \multicolumn{6}{|l|}{ Faixa Etária } \\
\hline 60 a 64 & 870 & 728 & 569 & 224 & 2391 \\
\hline 65 a 69 & 714 & 662 & 475 & 104 & 1955 \\
\hline 70 a 74 & 664 & 592 & 471 & 94 & 1821 \\
\hline 75 a 79 & 474 & 551 & 440 & 51 & 1516 \\
\hline 80 e mais & 702 & 740 & 599 & 69 & 2110 \\
\hline \multicolumn{6}{|l|}{ Sexo } \\
\hline Feminino & 1693 & 1840 & 1548 & 182 & 5263 \\
\hline Masculino & 1731 & 1433 & 1006 & 360 & 4530 \\
\hline \multicolumn{6}{|l|}{ Classificação do Caso } \\
\hline Intoxicação & 1967 & 3218 & 1859 & 528 & 7572 \\
\hline Efeito Adverso & 1457 & 55 & 695 & 14 & 2221 \\
\hline \multicolumn{6}{|l|}{ Circunstância da Intoxicação } \\
\hline Ignorada & 123 & 3202 & 12 & 521 & 3858 \\
\hline Acidental & 455 & 8 & 847 & 1 & 1311 \\
\hline Auto-Intoxicação & 662 & 5 & 515 & 1 & 1183 \\
\hline Não Determinada & 632 & 3 & 464 & 5 & 1104 \\
\hline Agressão & 95 & - & 21 & - & 116 \\
\hline \multicolumn{6}{|l|}{ Classe Terapêutica } \\
\hline $\begin{array}{l}\text { Outras drogas e as não especificadas (T50.9, T88.7, X44, X64, Y14, Y57.8, } \\
\text { Y57.9) }\end{array}$ & 784 & 827 & 708 & 32 & 2351 \\
\hline Antibióticos sistêmicos $(\mathrm{T} 36, \mathrm{Y} 40)$ & 39 & 1062 & 524 & 43 & 1668 \\
\hline $\begin{array}{l}\text { Antiepilépticos, sedativos, hipnóticos, antiparkinsonianos, drogas } \\
\text { psicotrópicas e psicoativas (F13.0, F19.0, T42, T43, X41, X61, Y11, Y46, } \\
\text { Y47, Y49) }\end{array}$ & 456 & 363 & 532 & 236 & 1587 \\
\hline $\begin{array}{l}\text { Analgésicos, antitérmicos e anti-reumáticos não opiáceos (T39, X40, } \\
X 60, Y 10, Y 45)\end{array}$ & 871 & 38 & 39 & 5 & 953 \\
\hline $\begin{array}{l}\text { Substâncias que atuam primariamente sobre o aparelho circulatório e } \\
\text { sobre o aparelho cardiovascular (T46, Y52) }\end{array}$ & 45 & 246 & 409 & 37 & 737 \\
\hline $\begin{array}{l}\text { Drogas que afetam principalmente o sistema nervoso autônomo ( } T 44 \text {, } \\
X 43, X 63, Y 13, Y 51)\end{array}$ & 465 & 60 & 34 & 8 & 567 \\
\hline $\begin{array}{l}\text { Substâncias de ação essencialmente sistêmica e substâncias } \\
\text { hematológicas (T45,Y43,Y44) }\end{array}$ & 183 & 107 & 117 & 10 & 417 \\
\hline Outros não classificados acima & 581 & 570 & 191 & 171 & 1513 \\
\hline Total & 3424 & 3273 & 2554 & 542 & 9793 \\
\hline 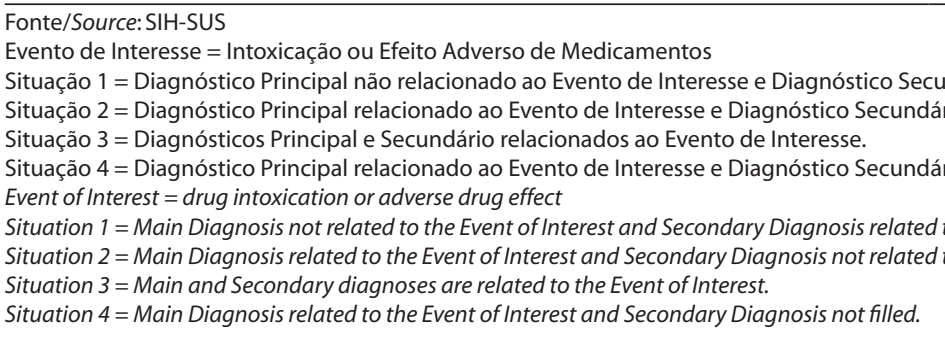 & $\begin{array}{l}\text { undário rel } \\
\text { rio não rel } \\
\text { rio não pr } \\
\text { to the Ever } \\
\text { to the Ever }\end{array}$ & $\begin{array}{l}\text { ionado ao } \\
\text { ionado ao } \\
\text { chido. } \\
\text { finterest. } \\
\text { finterest. }\end{array}$ & $\begin{array}{l}\text { ento de In } \\
\text { ento de In }\end{array}$ & asse. & \\
\hline
\end{tabular}


Tabela 2 - Casos e percentuais associados aos Diagnósticos Principal e Secundário relacionados às Situações 1 e 2. Brasil, 2004 a 2008.

Table 2 - Cases and percentage associated to main and secondary diagnosis related to situations 1 and 2. Brazil, 2004 to 2008.

\begin{tabular}{|c|c|c|}
\hline Diagnóstico Principal relacionado à Situação 1 & Casos & $\%$ \\
\hline S00-T14 Traumatismos em geral & 1363 & 39,8 \\
\hline T51-T65 Efeitos tóxicos substâncias origem predominantemente não medicinal & 734 & 21,4 \\
\hline T80-T88 Complicações cuidados médicos e cirúrgicos não classificados & 591 & 17,3 \\
\hline C00-C97 Neoplasias [tumores] malignas(os)s & 166 & 4,8 \\
\hline T66-T78 Outros efeitos de causas externas e os não especificados & 163 & 4,8 \\
\hline I00-199 Doenças do aparelho circulatório & 132 & 3,9 \\
\hline T20-T32 Queimaduras e corrosões & 39 & 1,1 \\
\hline J00-J99 Doenças do aparelho respiratório & 33 & 1,0 \\
\hline T15-T19 Efeito da penetração de corpo estranho através de orifício natural & 30 & 0,9 \\
\hline T40-Intoxicação por narcóticos e psicodislépticos (exceto T40.2, T40.3, T40.4) & 28 & 0,8 \\
\hline T90-T98 Sequelas de traumatismos, de intoxicações e de outras consequências & 22 & 0,6 \\
\hline L00-L99 Doenças da pele e do tecido subcutâneo & 18 & 0,5 \\
\hline N00-N99 Doenças do aparelho geniturinário & 15 & 0,4 \\
\hline K00-K93 Doenças do aparelho digestivo & 14 & 0,4 \\
\hline R00-R99 Sintomas, sinais e achados anormais de exames clínicos e laboratoriais & 14 & 0,4 \\
\hline Outros & 62 & 1,8 \\
\hline Total & 3424 & 100,0 \\
\hline Diagnóstico Secundário relacionado à Situação 2 & Casos & $\%$ \\
\hline W00-W19 Quedas & 1082 & 33,1 \\
\hline X58-X59 Exposição acidental a outros fatores e aos não especificados & 317 & 9,7 \\
\hline Y10-Y34 Eventos cuja intenção é indeterminada (exceção aos medicamentos) & 262 & 8,0 \\
\hline X86-Y09 Agressões & 231 & 7,1 \\
\hline V01-V99 Acidentes de transporte & 179 & 5,5 \\
\hline X40-X49 Env. ou intox. acid. e por exp. subst. nocivas (exceção medicamentos) & 169 & 5,2 \\
\hline Y40-Y84 Complicações de assistência médica e cirúrgica & 144 & 4,4 \\
\hline W20-W64 Exposição a forças mecânicas & 142 & 4,3 \\
\hline W85-W99 Exp. Corr. elétrica, radiação e às temperaturas e pressões extremas & 135 & 4,1 \\
\hline X60-X84 Lesões auto-provocadas intencionalmente (exceção medicamentos) & 112 & 3,4 \\
\hline Y85-Y89 Sequelas de causas externas de morbidade e mortalidade & 109 & 3,3 \\
\hline Y90-Y98 Fatores supl. relacionados com causas de morbidade e mortalidade & 101 & 3,1 \\
\hline X20-X29 Contato com animais e plantas venenosos & 88 & 2,7 \\
\hline X00-X09 Exposição ao fumo, ao fogo e às chamas & 68 & 2,1 \\
\hline X10-X19 Contato com uma fonte de calor ou com substâncias quentes & 52 & 1,6 \\
\hline Outros & 82 & 2,5 \\
\hline Total & 3273 & 100,0 \\
\hline
\end{tabular}

Fonte/Source: SIH-SUS

Evento de Interesse = Intoxicação ou Efeito Adverso de Medicamentos

Situação 1 = Diagnóstico Principal não relacionado ao Evento de Interesse e Diagnóstico Secundário relacionado ao Evento de Interesse.

Situação 2 = Diagnóstico Principal relacionado ao Evento de Interesse e Diagnóstico Secundário não relacionado ao Evento de Interesse.

Event of Interest = drug intoxication or adverse effect

Situation $1=$ Main Diagnosis not related to the Event of Interest and Secondary Diagnosis related to the Event of Interest.

Situation 2 = Main Diagnosis related to the Event of Interest and Secondary Diagnosis not related to the Event of Interest. 


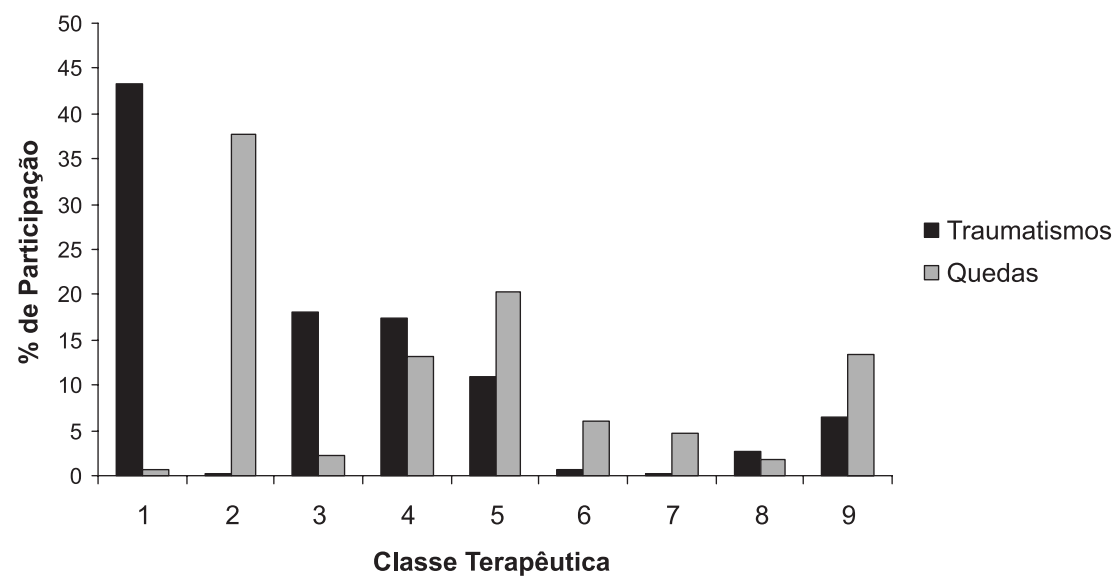

Fonte/Source: SIH-SUS

1 - Analgésicos, antitérmicos e anti-reumáticos não opiáceos (T39, X40, X60, Y10, Y45).

2 - Antibióticos sistêmicos (T36, Y40).

3 - Hormônios, seus substitutos sintéticos e seus antagonistas (T38,Y42).

4 - Antiepilépticos, sedativos, hipnóticos, antiparkinsonianos, drogas psicotrópicas e psicoativas (F13.0, F19.0,T42, $\mathrm{T} 43, \mathrm{X} 41, \mathrm{X} 61, \mathrm{Y} 11, \mathrm{Y} 46, \mathrm{Y} 47, \mathrm{Y} 49$ ).

5 - Outras drogas e as não especificadas (T50.9, T88.7, X44, $\mathrm{X} 64, \mathrm{Y} 14, \mathrm{Y} 57.8, \mathrm{Y} 57.9)$.

6 - Substâncias que atuam primariamente sobre o aparelho circulatório e sobre o aparelho cardiovascular (T46, Y52).

7 - Substâncias de ação no trato gastrointestinal $(T 47, Y 53)$.

8 - Drogas que afetam principalmente o sistema nervoso autônomo (T44,X43, X63, Y13, Y51).

9 - Outros não classificados acima (T37, Y41,T45, Y43, Y44, T48, Y55, T49, Y56, T41, Y48, X85, Y500, Y542, Y545, Y549, Y573, Y576)
1 - Nonopioid analgesics, antipyretics and antirheumatics (T39, X40, X60, Y10, Y45).

2 - Systemic antibiotics (T36, Y40).

3 - Hormones, their synthetic substitutes and antagonists (T38, Y42).

4 - Antiepileptics, sedative, hypnotic, antiparkinsonian, psychotropic and psychoactive drugs (F13.0, F19.0, T42, T43, X41, X61, Y11, Y46, Y47, Y49).

5 - Other and unspecified drugs (T50.9, T88.7, X44, X64,Y14, Y57.8, Y57.9).

6 - Substances that act primarily on the circulatory system and on the cardiovascular system (T46, Y52).

7- Substances of action in the gastrointestinal system (T47, Y53).

8 -Drugs primarily affecting autonomic nervous system (T44, $X 43, X 63, Y 13, Y 51)$.

9- Other not classified above (T37, Y41,T45, Y43, Y44, T48, Y55, $T 49, Y 56, T 41, Y 48, X 85, Y 500, Y 542, Y 545, Y 549, Y 573, Y 576)$

Figura 2 - Percentual de participação das classes terapêuticas nos "Traumatismos em Geral" e nas “Quedas”. Brasil, 2004 a 2008.

Figure 2 - Percentage participation of therapeutic classes in "Injuries in general" and "Falls". Brazil, 2004 to 2008.

foram responsáveis por $37 \%$ das internações relacionadas a auto-intoxicação. A circunstância agressão tem $83 \%$ de seus casos relacionados a outras substâncias não classificadas, onde o código X85 ("agressão por meio de drogas, medicamentos e substâncias biológicas") é contemplado. Há diferenças entre a distribuição percentual das classes terapêuticas envolvidas com as categorias "não determinada" e "ignorada". Comparativamente, os antibióticos sistêmicos se destacam para a categoria "ignorada", enquanto que os analgésicos, antitérmicos e antirreumáticos não opiáceos, bem como os fármacos que afetam principalmente o sistema nervoso autônomo, apresentam maior concentração para a categoria "não determinada".
Ao comparar as 4 situações, o teste de Kruskal-Wallis apresentou diferenças significativas entre as idades (estatística do teste $=116,769, \mathrm{p}$-valor $=0,0$ ) e o tempo de permanência no hospital (estatística do teste $=99,7888, p$-valor $=0,0$ ). A situação 4 apresentou a menor média de idade, 68,9 anos, seguida pelas situações 1, 2 e 3, com médias de 71,8; 72,6 e 72,8, respectivamente; e o maior tempo médio de permanência, 7,3 dias. As demais situações apresentaram tempo médio bem inferior, em torno de 4,5 dias. A situação 4 foi a que apresentou a maior proporção de casos de intoxicação relacionados a transtornos mentais e comportamentais $(55,4 \%)$, sendo a maioria desses relacionados a sedativos, hipnóticos e outras substâncias psicoativas, F11.0 = uso de opiáceos (67); 

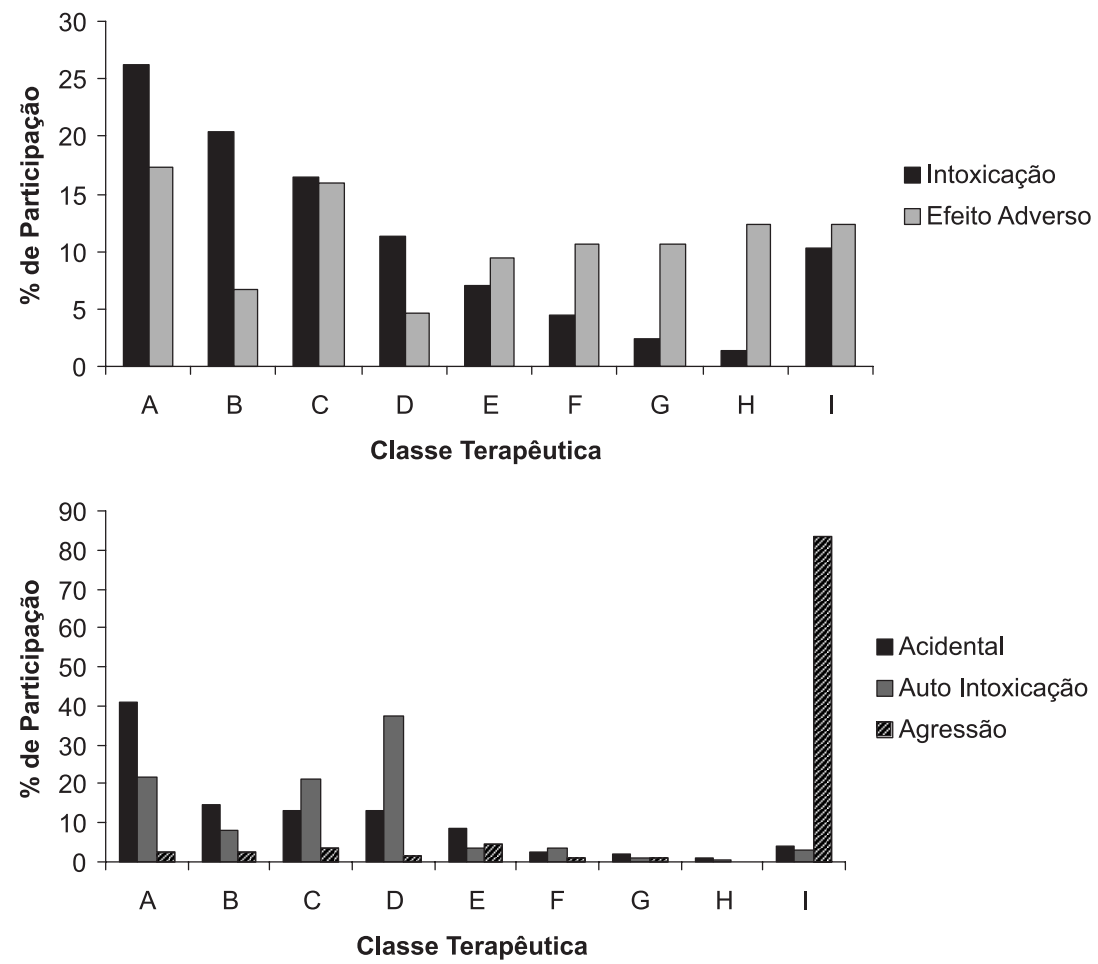

Acidental

$\square$ Auto Intoxicação

\Agressão

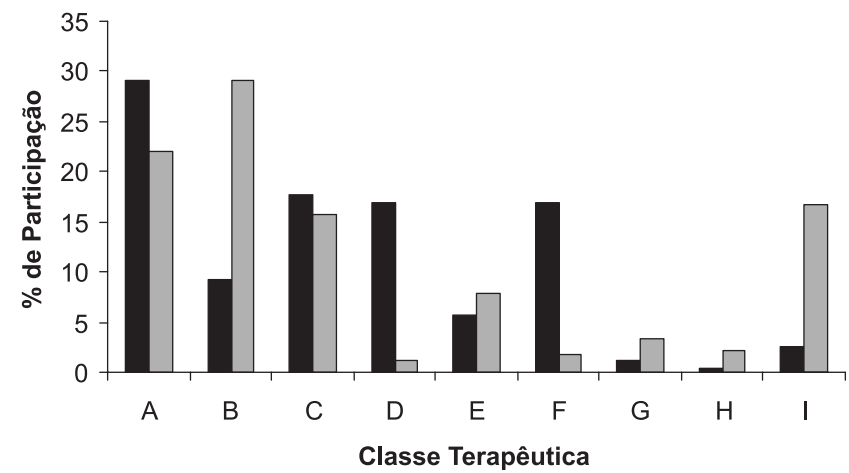

Não Determinada $\square$ Ignorada

Fonte/Source: SIH-SUS

A - Outras drogas e as não especificadas (T50.9, T88.7, X44, X64, Y14, Y57.8, Y57.9)

B - Antibióticos sistêmicos (T36,Y40)

C - Antiepilépticos, sedativos, hipnóticos, antiparkinsonianos, drogas psicotrópicas e psicoativas (F13.0, F19.0, T42, $\mathrm{T} 43, \mathrm{X} 41, \mathrm{X} 61, \mathrm{Y} 11, \mathrm{Y} 46, \mathrm{Y} 47, \mathrm{Y} 49)$

$D$ - Analgésicos, antitérmicos e anti-reumáticos não opiáceos (T39, X40, X60, Y10, Y45)

E - Substâncias que atuam primariamente sobre o aparelho circulatório e sobre o aparelho cardiovascular (T46,Y52)

$\mathrm{F}$ - Drogas que afetam principalmente o sistema nervoso autônomo (T44, X43, X63, Y13,Y51)

G - Substâncias de ação essencialmente sistêmica e substâncias hematológicas (T45, Y43, Y44)

H - Hormônios, seus substitutos sintéticos e seus antagonistas $(\mathrm{T} 38, \mathrm{Y} 42)$

I - Outros não classificados acima
A - Other and unspecified drugs ((T50.9, T88.7, X44, X64,Y14, Y57.8, Y57.9)

B - Systemic antibiotics (T36, Y40)

C - Antiepileptic, sedative-hypnotic, antiparkinsonism drugs, psychotropic and psychoactive drugs (F13.0, F19.0, T42, T43, X41,X61, Y11, Y46, Y47, Y49)

$D$ - Nonopioid analgesics, antipyretics and antirheumatics (T39, X40, X60, Y10, Y45)

$E$ - Substances that act primarily on the circulatory system and on the cardiovascular system (T46, Y52)

F-Drugs primarily affecting autonomic nervous system (T44, $X 43, X 63, Y 13, Y 51)$

$G$ - Substances of action primarily systemic and haematological substances $(T 45, Y 43, Y 44)$

H - Hormones, their synthetic substitutes and antagonists (T38, Y42).

1- Other not classified above.

Figura 3 - Percentual de participação das Classes Terapêuticas na Intoxicação e no Efeito Adverso e nas categorias da Circunstância da Intoxicação. Brasil, 2004 a 2008.

Figure 3 - Percentage of participation in therapeutic class Intoxication and Adverse Effect and the categories of Intoxication Cause. Brazil, 2004 to 2008. 
F13.0 = uso de sedativos e hipnóticos (81); F15.0 = uso de outros estimulantes, inclusive a cafeína (37); F19.0 = uso de múltiplas drogas e de outras substâncias psicoativas (115).

A comparação das circunstâncias da intoxicação pelo teste de Kruskal-Wallis também apresentou diferenças significativas entre as idades (estatística do teste $=19,577, \mathrm{p}$-valor $=0,00149995)$ e o tempo de permanência no hospital (estatística do teste $=55,2633$, $p$-valor $=1,15228 \mathrm{E}-10)$. A circunstância acidental apresentou a maior média de idade $(72,9)$, sendo o menor tempo médio de permanência observado para a circunstância autointoxicação (4,1 dias).

Ao comparar os sexos, o teste de Wilcoxon-Mann-Whitney concluiu pela hipótese de que a mediana da idade é maior para os pacientes idosos do sexo feminino (72 contra 70, p-valor $=0,00$ ). Apesar de as medianas do tempo de permanência no hospital serem iguais para os dois sexos, 3 dias, esse teste, por ser construído com base nas médias dos postos, concluiu pela hipótese de que a mediana do tempo é maior para os pacientes do sexo masculino ( $\mathrm{p}$-valor $=0,000650428$ ).

Ao comparar os casos classificados em "intoxicação" e "efeito adverso", o teste de Wilcoxon-Mann-Whitney concluiu pela hipótese de que a mediana da idade para os casos de intoxicação e efeito adverso são iguais ( $\mathrm{p}$-valor $=0,189475)$. Porém, a mediana do tempo de permanência no hospital é maior para os casos de intoxicação em comparação com os de efeito adverso, apesar de apresentarem a mesma mediana (3,0 dias) $(\mathrm{p}$-valor $=0,000141697)$.

\section{Discussão}

Enquanto o total de internações hospitalares registradas no país pelo SIH-SUS diminuiu $7 \%$ no período de 2004 a $2008^{50}$, as internações hospitalares de idosos diminuíram $3 \%$ e as relacionadas a intoxicações e efeitos adversos de medicamentos aumentaram quase $14 \%$, chamando a atenção da importância desses agravos para essa população.
O aumento com a idade do risco de sofrer internação hospitalar decorrente de intoxicação e efeito adverso de medicamentos também foi observado por Burgess et al. ${ }^{23} \mathrm{e}$ Loyola Filho et al. ${ }^{35}$ para reações adversas. Esses resultados estão em consonância com os estudos que afirmam que o consumo de medicamentos aumenta com o avanço da idade $^{8-10}$.

A comparação com os dados do SINITOX ${ }^{15}$ é prejudicada, uma vez que este apresenta casos de intoxicação de modo geral, podendo ou não terem ocasionado internação hospitalar. Dados desse sistema para o período do estudo apontam para os seguintes percentuais de participação dos idosos nas intoxicações por medicamentos decrescentes segundo a faixa etária: $1,41 \%$ (60 a 69 anos), 0,81\% (70 a 79 anos) e 0,48\% (80 anos e mais). Já os coeficientes de morbidade por 100 mil habitantes mostraram-se crescentes, 0,45, 0,46 e 0,64, respectivamente, vindo a reiterar que entre os idosos há uma elevação do risco de intoxicação com o aumento da faixa etária.

Coelho Filho et al. ${ }^{12}$, ao estudarem o perfil de utilização de medicamentos por idosos, observaram que o maior número médio de medicamentos prescritos em uso ocorre no sexo feminino e com idade de 75 anos ou mais. Loyola Filho et al., também em um estudo similar, verificaram que a associação entre sexo feminino e consumo de medicamentos prescritos persistiu mesmo após ajustamento por consultas ao médico e estado de saúde. Observaram também aumento no consumo de medicamentos prescritos com a idade. Estes resultados podem explicar a inversão por sexo observada a partir de 75 anos de idade nas taxas de internação hospitalar.

$\mathrm{O}$ fato de a mediana da idade do sexo feminino ser superior ao da idade do sexo masculino é uma conseqüência da maior longevidade das mulheres. Já o fato de o tempo mediano de internação do sexo masculino ser superior ao do sexo feminino pode estar relacionado à maior gravidade dos casos, à pior condição do estado de saúde, a comorbidades pré-existentes e, em 
especial, a questões comportamentais específicas do gênero homem, que dependem de fatores culturais e, de uma maneira geral, sociais, tais como comportamento frente à doença, à procura por serviços de saúde, ao hábito de fumar, ao alcoolismo, ao tipo de dieta, ao ambiente de trabalho, à atividade física, ao peso corporal, entre outros ${ }^{51}$.

$\mathrm{Na}$ busca pelas principais classes terapêuticas envolvidas nas internações de idosos, surge em primeiro lugar a categoria "outras drogas e as não especificadas". O que deveria ser uma exceção, sendo assinalada somente em algumas situações, no Brasil vira regra, com quase um quarto das observações. Lessa et al. $^{37}$, ao estudarem internações de crianças menores de 1 ano, também se depararam com essa categoria envolvida com a maioria dos casos. É preciso salientar que o código da CID-10 que concentra a maior parte dessas observações é o T50.9, sendo a categoria T50 referente a intoxicação por diuréticos e outras drogas, medicamentos e substâncias biológicas e as não especificadas. Assim, estudos que não consideram o quarto dígito podem estar superestimando os casos relacionados a diuréticos, criando vieses importantes que devem ser observados com maior rigor pelos programas de farmacovigilância. Visando à melhoria da qualidade dos dados com a diminuição de casos assinalados como T50.9, recomenda-se o treinamento das equipes responsáveis pelo preenchimento da Autorização de Internação Hospitalar (AIH), bem como sua sensibilização acerca da importância dessa informação detalhada para os programas de farmacovigilância. Contudo, sabe-se que muitas vezes a deficiência da informação é proveniente do relato do paciente e de seus familiares.

Em segundo lugar, aparecem os antibióticos sistêmicos. A grande participação desses medicamentos nas internações de idosos (17\%), em especial nas relacionadas às situações 2 (32\%) e 3 (21\%), não foi encontrada nos estudos internacionais analisados. O que se observou em trabalhos realizados nos Estados Unidos ${ }^{27}$ sobre eventos adversos, e na Holanda ${ }^{21}$, Itália ${ }^{25}$, Espanha ${ }^{17}$,
Inglaterra ${ }^{18}$ e Austrália ${ }^{23}$ sobre reações adversas foram percentuais bem menores: $3,8 \%^{27}, 4,1 \%^{21}, 5,7 \%^{25}, 6,3 \%^{17}$ e $9,0 \%{ }^{18,23}$, indicando uma diferença importante, que pode vir a ser explicada pelo maior controle desses países na venda desses produtos. No Brasil, no período do estudo, ocorria a comercialização sem receita de medicamentos que deveriam ser vendidos apenas sob prescrição ${ }^{52,53}$. Entre eles figuravam os antibióticos, que somente em maio de 2011 , com a adoção da Resolução n ${ }^{\circ} 20$ da Agência Nacional deVigilância Sanitária (ANVISA) ${ }^{54}$, começaram a ser comercializados com a retenção da receita. Essa medida representa um passo importante na luta pelo uso racional de medicamentos, pois além de coibir a automedicação, permite a supervisão da prescrição por parte do farmacêutico, provendo também orientações ao consumidor sobre o uso correto do medicamento. Novos estudos devem ser realizados para avaliar o impacto dessa legislação nas intoxicações e efeitos adversos de idosos.

Em terceiro lugar, surgem os psicotrópicos, psicoativos, antiepilépticos, sedativos, hipnóticos e antiparkinsonianos com 16,2\% de participação. A alta prevalência de desordens neurodegenerativas, como doença de Parkinson e Alzheimer, e desordens do humor, como depressão, insônia e ansiedade, podem explicar o uso desses medicamentos por essa faixa da população ${ }^{5}$. Assim, a alta participação desses medicamentos na situação $4(43,5 \%)$ pode ser explicada pela prevalência de transtornos mentais e comportamentais nela observados. Contudo, alguns desses medicamentos são potencialmente contraindicados para idosos ${ }^{55-57}$.

As classes terapêuticas de amplo uso (analgésicos, antitérmicos e antirreumáticos não opiáceos ${ }^{52,58}$ ) e a mais prescrita (medicamentos com ação cardiovascu$\operatorname{lar}^{9-12,55,59-61}$ ) não se apresentaram como as mais envolvidas nas internações decorrentes de intoxicações e efeitos adversos de medicamentos, ocupando a quarta e quinta posição, respectivamente.

A separação das internações em 4 situações possibilitou traçar um perfil de cada 
uma delas, o que pode favorecer as análises e direcionar estudos e medidas de controle específicas. A situação 1 se caracteriza por apresentar maior proporção de efeitos adversos que as demais, especialidade do atendimento concentrada em clínica e cirúrgica, maior população masculina, principal classe terapêutica dada pelos analgésicos, antitérmicos e anti-reumáticos não opiáceos e traumatismos, é o seu principal agravo relacionado. A situação 2 se caracteriza por intoxicações, especialidade do atendimento concentrado em clínica, maior população feminina, principal classe terapêutica dada pelos antibióticos sistêmicos e quedas constituindo seu principal agravo relacionado. A situação 3 se caracteriza por intoxicações, especialidade do atendimento concentrada em clínica, maior população feminina, principal classe terapêutica dada pelas outras drogas e as não especificadas. A situação 4 se caracterizae por apresentar maior proporção de intoxicações, concentração de transtornos mentais e comportamentais, especialidade do atendimento dividida em clínica e psiquiátrica, maior população masculina, menor idade média, maior média de permanência no hospital, principal classe terapêutica dada pelos psicotrópicos, psicoativos, antiepilépticos, sedativos, hipnóticos e antiparkinsonianos.

Essa metodologia também permitiu a identificação dos principais agravos relacionados a intoxicações e efeitos adversos de medicamentos. Traumatismos e quedas foram identificados como os principais agravos relacionados a esses eventos, o que está de acordo com os resultados obtidos por Hamra et al. ${ }^{6}$, que sugerem que os pacientes que fazem uso de medicamentos possuem maior risco de sofrerem fratura por queda.

No caso dos traumatismos, estes estavam relacionados ao diagnóstico principal e à intoxicação ou efeito adverso de medicamentos ao diagnóstico secundário, o que corresponde à situação 1. Os medicamentos mais envolvidos nessas internações foram os analgésicos, antitérmicos e antirreumáticos não opiáceos, respondendo por quase $45 \%$ dos casos. Nessa situação, como a intoxicação ou o efeito adverso não foram a causa principal da internação, parte desses casos podem ter ocorrido em ambiente hospitalar. Apesar de a base de dados do estudo não permitir a inferência do número exato dessas ocorrências, acredita-se que a análise da situação 1 possa indicar alguns dos problemas causados por medicamentos ao paciente idoso no ambiente hospitalar. Nesse sentido, deve-se promover uma revisão do uso de analgésicos, antitérmicos e antirreumáticos não opiáceos em pacientes idosos traumatizados de forma a evitar agravos advindos da polifarmácia, da prescrição errônea e da iatrogenia medicamentosa.

Nos casos das quedas, a intoxicação e/ ou efeito adverso de medicamentos estava associado ao diagnóstico principal e a queda ao diagnóstico secundário, o que corresponde à situação 2. Nestas internações surgem como principais classes terapêuticas envolvidas os antibióticos sistêmicos, contrariando os estudos que apontam os psicotrópicos como os principais medicamentos envolvidos nesses eventos ${ }^{5,62-64}$.

A relação entre quedas e antibióticos foi também assinalada por Lessa et al. ${ }^{37}$ ao estudar internações de crianças menores de um ano decorrentes de intoxicações e efeitos adversos de medicamentos. Uma possível explicação pode vir do trabalho publicado recentemente por Wright et al. ${ }^{65}$, no qual afirmam que em pacientes idosos tratados com bloqueadores dos canais de cálcio, o uso dos antibióticos eritromicina ou claritromicina foi associado a um aumento do risco de hipotensão ou choque requerendo hospitalização.

O trabalho apresenta limitações comuns aos estudos baseados em dados secundários provenientes do SIH-SUS ${ }^{35}$. Em especial, para o presente estudo, o grande uso dos dígitos 8 (outros) e 9 (não especificados) para o quarto dígito dos códigos do capítulo 19 (T36 a T50) e do capítulo 20 (Y40 a Y57) impediu o detalhamento de $57 \%$ dos medicamentos dentro de uma classe terapêutica. As diferentes metodologias adotadas nos vários estudos internacionais existentes 
para mensurar as internações hospitalares decorrentes de reações adversas ${ }^{16-25}$ e eventos adversos ${ }^{26-29}$ de medicamentos dificultam o confronto com os resultados desse estudo. Alguns desses trabalhos ${ }^{16,18}$ consideraram como reação adversa casos que apresentassem no diagnóstico principal códigos da CID-10 que incluíssem as palavras "induzido por" ou "devido a" medicamentos, ficando para o diagnóstico secundário o detalhamento da classe terapêutica com códigos previstos nesse estudo. Como nem sempre há um bom preenchimento do diagnóstico secundário, pode ter ocorrido subestimação de casos de efeito adverso. Apesar da metodologia adotada com a separação nas 4 situações, não foi possível distinguir, dentre os casos da situação 1 , aqueles em que a intoxicação ou efeito adverso estavam presentes no momento da admissão hospitalar daqueles em que esses eventos ocorreram durante a internação. Contudo, diferentemente do trabalho de Rozenfeld ${ }^{36}$, foi possível chegar a uma amostra mais próxima dos eventos que ocorrem no ambiente hospitalar, o que permite sugerir maior vigilância ao processo de utilização de analgésicos nesse local. Stausberg et al. ${ }^{32}$ utilizaram um procedimento semelhante ao considerar o diagnóstico secundário como uma "proxy" para reações adversas adquiridas no hospital.

\section{Conclusão}

Os resultados encontrados refletem a tendência crescente dos problemas associados ao uso de medicamentos por idosos. Verificou-se que o perfil de consumo não é suficiente para explicar as concentrações de casos nas principais classes terapêuticas. $\mathrm{Na}$ busca pelas causas, sugere-se a adoção de programas mais efetivos de farmacovigilância capazes de monitorar as diferentes etapas do processo de uso de medicamentos: prescrição, dispensação, comercialização, administração e adesão ao tratamento.

\section{Referências}

1. Brasil. Ministério da Saúde. Estatuto do Idoso. Série E.

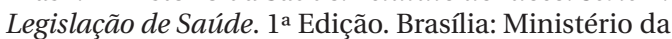
Saúde; 2003. 70 p.

2. Departamento de Informática do SUS (Datasus). Informações de Saúde. Demográficas e Socioeconômicas. População Residente. Disponível em: http://tabnet. datasus.gov.br/cgi/ deftohtm.exe?ibge/cnv/popuf.def [Acessado em 08 de fevereiro de 2012].

3. Kalache A, Veras RP, Ramos RL. O envelhecimento da população mundial. Um desafio novo. Rev Saúde Pública 1987; 21(3): 200-10.

4. Chaimowicz F. A saúde dos idosos às vésperas do século XXI: problemas, projeções e alternativas. Rev Saúde Pública 1997; 31(2): 184-200.

5. Chaimowicz F, Ferreira TJXM, Miguel DFA. Use of psychoative drugs and related falls among older people living in a community in Brazil. Rev Saúde Pública 2000; 34(6): 631-35.

6. Hamra A, Ribeiro MB, Miguel OF. Correlação entre fratura por queda em idosos e uso prévio de medicamentos. Acta Ortop Bras 2007; 15(3): 143-5.

7. Flores VB, Benvegnú, LA. Perfil de utilização de medicamentos em idosos da zona urbana de Santa
Rosa, Rio Grande do Sul, Brasil. Cad Saúde Pública 2008; 24(6): 1439-46.

8. Bertoldi AD, Barros AJD, Hallal PC, Lima RC. Utilização de medicamentos em adultos: prevalência e determinantes individuais. Rev Saúde Pública 2004; 38(2): 228-38.

9. Loyola Filho AI, Uchoa E, Firmo JOA, Lima-Costa MF. Estudo de base populacional sobre o consumo de medicamentos entre idosos: Projeto Bambuí. Cad Saúde Pública 2005; 21(12): 545-53.

10. Loyola Filho AI, Uchoa E, Lima-Costa MF. Estudo epidemiológico de base populacional sobre uso de medicamentos entre idosos na Região Metropolitana de Belo Horizonte, Minas Gerais, Brasil. Cad Saúde Pública 2006; 22(12): 2657-67.

11. Flores LM, Mengue SS. Uso de medicamentos por idosos em região do sul do Brasil. Rev Saúde Pública 2005; 39(6): 924-9.

12. Coelho Filho JM, Marcopito LF, Castelo A. Perfil de utilização de medicamentos por idosos em área urbana do Noroeste do Brasil. Rev Saúde Pública 2004; 38(4): 557-64. 
13. Bernardes ACA, Chorilli M, Oshima-Franco Y. Intoxicação Medicamentosa no Idoso. Saúde em Revista 2005; 7(15): 53-61.

14. Teixeira JJV, Lefevre F. A prescrição medicamentosa sob a ótica do paciente idoso. Rev Saúde Pública 2001; 35(2): 207-13.

15. Fundação Oswaldo Cruz. Instituto de Comunicação e Informação Científica e Tecnológica em Saúde. Sistema Nacional de Informações Tóxico-Farmacológicas SINITOX. Registros de Intoxicações. Dados Nacionais. 1999 - 2009. Disponível em: http:/ /www.fiocruz.br/ sinitox_novo/cgi/cgilua.exe/sys/start.htm?sid=8 [Acessado em 03 de fevereiro de2012].

16. Brvar M, Fokter N, Bunc M, Mozina M. The frequency of adverse drug reaction related admissions according to method of detection, admissions according to method of detection, admission urgency and medical department specialty. BMC Clinical Pharmacology 2009; 9:8.

17. Carrasco-Garrido P, Andrés, LA, Barrera VH, Miguel GA, Jiménez-García R. Trends of adverse drug reactions related-hospitalizations in Spain (2001-2006). BMC Health Serv Res 2010; 10: 287.

18. Patel H, Bell D, Molokhia M. Trends in hospital admissions for adverse drug reactions in England: analysis of national hospital episode statistics 19982005. BMC Clin Pharmacol 2007; 7:9.

19. Pirmohamed M, James S, Meakin S, Green C, Scott AK, Walley TJ et al. Adverse drug reactions as cause of admission to hospital: prospective analysis of 18820 patients. BMJ 2004; 329: 15.

20. Van der Hoof CS, Sturkenboom CJM, Van Grootheest $\mathrm{K}$, Kingma HJ, Stricker BHCh. Adverse drug reactionrelated hospitalisations. Drug Safety 2006; 29(2): 161-8.

21. Van der Hoof CS, Dieleman JP, Siemes C, Aarnoudse A-JLHJ, Verhamme KMC, Stricker BHCH et al. Adverse drug reaction-related hospitalizations: a populationbased cohort study. Pharmacoepidemiol Drug Safety 2008; 17: 365-71.

22. Beijer HJM, Blaey CJ. Hospitalisation caused by adverse drug reactions (ADR): a meta-analysis of observational studies. Pharm World Sci 2002; 24(2): 46-54.

23. Burgess CL, Holman CDJ, Satti AG. Adverse drug reactions in older Australians, 1981-2002. MJA 2005; 182: 267-70.

24. Hartholt KA, Van der Velde N, Looman CWN, Panneman MJM, Van Beeck EF, Patka P, Van der Cammen TJM. Adverse drug reactions related hospital admissions in persons aged 60 years and over, the Netherlands, 19812007: less rapid increase, different drugs. PLoS One 2010; 5(11): 13.977 .

25. Onder G, Pedone C, Landi F, Cesari M, Vedova CD, Bernabei R. Adverse drug reactions as cause of hospital admissions: result from the Italian Group of Pharmacoepidemiology in the Elderly (GIFA). J Am Ger Soc 2002; 50(12): 1962-8.
26. Queneau P, Bannwarth B, Carpentier F, Guliana JM, Bouget J, Trombert B et al. Emergence department visits caused by adverse drug events. Drug Safety 2007; 30(1): 81-8.

27. Budnitz DS, Pollock D, Weidenbach KN, Mendelsohn AB, Schroeder TJ, Annest JL. National surveillance of emergency department visits for outpatient adverse drug events. JAMA 2006; 296(15): 1858-66.

28. Budnitz DS, Shehab N, Kegler SR, Richards CL. Medication use leading to emergency department visits for adverse drug events in older adults. Ann Intern Med 2007; 147(11): 755-66.

29. Budnitz DS, Lovegrove MC, Shehab N, Chesley L, Richards CL. Emergency hospitalization for adverse drug events in older Americans. New Engl J Med 2007; 365: 2002-12.

30. Howard RL, Avery AJ, Slavenburg S, Royal S, Pipe G, Lucassen P, Pirmohamed M. Which drugs cause preventable admissions to hospital? A systematic review. Br J Clin Pharmacol 2006; 63(2): 136-47.

31. Juurlink DN, Mamdani M, Kopp A, Laupacis A, Redelmeier DA. Drug-Drug interactions among elderly patients hospitalized for drug toxicity. JAMA 2003; 289(13) 1652-8.

32. Stausberg J, Hasford J. Drug-related admissions and hospital-acquired adverse drug events in Germany: a longitudinal analysis from 2003 to 2007 of ICD-10-coded routine data. BMC Health Serv Res 2011; 11(134): 1-9.

33. Lebrão ML, Mello Jorge MH, Laurenti, R. II - Morbidade hospitalar por lesões e envenenamentos. Rev Saúde Pública 1997; 31(S4): 26-37.

34. Gawryszewski VP, Mello Jorge MHP, Koizumi MS. Mortes e internações por causas externas entre os idosos no Brasil: o desafio de integrar a saúde coletiva e atenção individual. Rev Assoc Méd Bras 2004; 50(1): 97-103.

35. Loyola Filho AI, Matos DL, Giatti L, Afradique ME, Peixoto SV, Lima-Costa MF. Causas de internações hospitalares entre idosos brasileiros no âmbito do Sistema Único de Saúde. Epidemiol Serv Saúde 2004; 13(4): 229-38.

36. Rozenfeld S. Agravos provocados por medicamentos em hospitais do Estado do Rio de Janeiro, Brasil. Rev Saúde Pública 2007; 41(1): 108-15.

37. Lessa MA, Bochner R. Análise das internações hospitalares de crianças menores de um ano relacionadas a intoxicações e efeitos adversos de medicamentos no Brasil. Rev Bras Epidemiol 2008; 11(4): 660-74.

38. Carvalho-Filho ET, Saporetti L, Souza MAR, Arantes ACLQ, Vaz MYKC, Hojaiji NHSL et al. Iatrogenia em pacientes idosos. Rev Saúde Pública 1998; 32: 36-42.

39. Passarelli MCG, Jacob-Filho W, Figueras A. Adverse drug reactions in an elderly hospitalised population: inappropriate prescription is a leading cause. Drugs Aging 2005; 22: 767-77. 
40. Departamento de Informática do SUS (Datasus). Movimento de Autorização de Internação Hospitalar. Arquivos Reduzidos, 2004. CD-ROM. Brasília: Ministério da Saúde.

41. Departamento de Informática do SUS (Datasus). Movimento de Autorização de Internação Hospitalar. Arquivos Reduzidos, 2005. CD-ROM. Brasília: Ministério da Saúde.

42. Departamento de Informática do SUS (Datasus). Movimento de Autorização de Internação Hospitalar. Arquivos Reduzidos, 2006. CD-ROM. Brasília: Ministério da Saúde.

43. Departamento de Informática do SUS (Datasus). Movimento de Autorização de Internação Hospitalar. Arquivos Reduzidos, 2007. CD-ROM. Brasília: Ministério da Saúde.

44. Departamento de Informática do SUS (Datasus). Movimento de Autorização de Internação Hospitalar. Arquivos Reduzidos, 2008. CD-ROM. Brasília: Ministério da Saúde.

45. Organização Mundial da Saúde. Ministério da Saúde. Universidade de São Paulo. Classificação Estatística Internacional de Doenças e Problemas Relacionados à Saúde. Décima Revisão. Volume 1. São Paulo: EDUSP; 2004.

46. World Health Organization. International Drug Monitoring: the role of the hospital. Geneva, Switzerland: World Health Organization; 1966. Technical Report Series No 425. Disponível em: http://whqlibdoc.who.int/trs/ WHO_TRS_425.pdf. [Acessado em 10 de janeiro de 2012].

47. Brasil. Ministério da Saúde. Secretaria Nacional de Assistência à Saúde. Secretaria de Assistência a Saúde. Portaria SAS No 743, de 22 de dezembro de 2005. Disponível em: http://dtr2004.saude.gov.br/dab/docs/ legislacao/portaria743_22_12_05.pdf. [Acessado em 16 de março de 2012].

48. Sistema de Informações Hospitalares do Sistema Único de Saúde (SIH-SUS): uma avaliação preliminar do seu desempenho no monitoramento da doença hemolítica perinatal Rh(D). Cad Saúde Pública 2008; 24(3): 606-14.

49. Callegari-Jacques SM. Bioestatística. Princípios e Aplicações. Porto Alegre: Artmed; 2003.

50. Brasil. Ministério da Saúde. Departamento de Informática do SUS (DATASUS). Informações de Saúde. Epidemiológicas e Morbidade. Morbidade Hospitalar do SUS. Disponível em: http:/ /www2.datasus.gov.br/ DATASUS/index.php?area=0203 [Acessado em $10 \mathrm{de}$ fevereiro de 2012].

51. Laurenti, R, Mello Jorge MHP, Gotlieb, SLD. Perfil epidemiológico da morbi-mortalidade masculina. Cien Saúde Colet 2005; 10(1): 35-46.

52. Bortolon PC, Medeiros EFF, Naves, JOS, Karnikowski MGO, Nóbrega OT. Análise do perfil de automedicação em mulheres idosas brasileiras. Cien Saúde Colet 2008; 13(4): 1219-26.
53. Berquó LS, Barros AJD, Lima RC, Bertoldi AD. Utilização de antimicrobianos em uma população urbana. Rev Saúde Pública 2004; 38(2): 239-46.

54. Agência Nacional de Vigilância Sanitária (ANVISA). Resolução da Diretoria Colegiada nº 20, de 5 de maio de 2011. Dispõe sobre o controle de medicamentos à base de substâncias classificadas como antimicrobiana, de uso sob prescrição médica, isoladas ou em associação. Diário Oficial da União; 2011.

55. Mosegui GBG, Rozenfeld S, Veras RP, Vianna CMM. Avaliação da qualidade do uso de medicamentos em idosos. Rev Saúde Pública 1999; 33(5): 437-44.

56. Fick DM, Cooper JW, Wade WE, Waller JL, Maclean R, Beers MH. Updating the Beers criteria for potentially inappropriate medication use in older adults. Arch Intern Med 2003; 8(22): 2716-24.

57. Huf, G, Lopes CS, Rozenfeld S. O uso prolongado de benzodiazepínicos em mulheres de um centro de convivência para idosos. Cad Saúde Pública 2000; 16(2): 351-62.

58. Tierling VL, Paulino MA, Fernandes LC, Schenkel EP, Mengue SS. Level of knowledge of the compositions of analgesic medication containing aspirin. Rev Saúde Pública 2004; 38(2): 223-7.

59. Oliveira CAP, Marin MJS, Marchioli M, Pizoletto, BHM, Santos, RV. Caracterização dos medicamentos prescritos aos idosos na Estratégia Saúde da Família. Cad Saúde Publica 2009; 25(5): 1007-16.

60. Ribeiro, AQ, Rozenfeld, S, Klein, CH, César, CC, Acurcio, FA. Inquérito sobre o uso de medicamentos por idosos aposentados, Belo Horizonte, MG. Rev Saúde Pública 2008; 42(4): 724-32.

61. Bueno CS, Oliveira KR, Berlezi EM, Eickhoff HM, Dallepiane, LB, Giradon-Perlini, NMO et al. Utilização de medicamentos e risco de interações medicamentosas em idosos atendidos pelo Programa de Atenção ao Idoso da Unijuí. Rev Ciênc Farm Básica Apl 2009; 30(3): 331-8.

62. Coutinho ESF, Silva SD. Uso de medicamentos como fator de risco para fratura grave decorrente de queda em idosos. Cad Saúde Pública 2002; 18(5): 1359-66.

63. Guimarães JMN, Farinatti PTV. Análise descritiva de variáveis teoricamente associadas ao risco de quedas em mulheres idosas. Rev Bras Med Esporte 2005; 11(5): 299-305.

64. Menezes RL, Bachion MM. Estudo da presença de fatores de riscos intrínsecos para quedas, em idosos institucionalizados. Cien Saúde Colet 2008; 13(4): 1209-18.

65. Wright AJ, Gomes T, Mamdani MM, Horn JR, Juurlink DN. The risk of hypotension following co-prescription of macrolide antibiotics and calcium-channel blockers. CMAJ 2011; 183(3): 303-7.

Recebido em: 01/09/11

Versão final apresentada em: 26/03/12

Aprovado em: 24/04/12 